\title{
Quantifying Collective Performance in Rugby Union
}

\section{Guillaume Saulière ${ }^{1 *}$, Jérôme Dedecker ${ }^{2}$, Issa Moussa ${ }^{1}$, Julien Schipman ${ }^{1}$, Jean-François Toussaint ${ }^{1,3}$ and Adrien Sedeaud ${ }^{1}$}

'IRMES, Institut de Recherche bio-Médicale et d'Epidémiologie du Sport, EA7329, INSEP \& Université Paris Descartes, Sorbonne Paris Cité, Paris, France, ${ }^{2}$ Laboratoire MAP5, Université Paris Descartes, Sorbonne Paris Cité, Paris, France,

${ }^{3}$ Centre d'Investigations en Médecine du Sport, Hôtel-Dieu, Assistance Publique - Hôpitaux de Paris, Paris, France

Objectives: The aim of this study was to quantify collective experience based on cumulative shared selections of players and to assess its impact on team performance in international rugby union. We assume that the greater the experience, the better the group will perform.

Methods: Scoresheets of all games involving at least one of all 10 nations participating at the Rugby Championship and the Six Nations Championship were collected from the end of the 1999 Rugby World Cup (RWC) up to the 2015 RWC. A single indicator quantifying the cumulative shared selections (CSS, the number of selections that each player has shared with the other ones) was computed for each match as a key collective experience indicator. The World Rugby Ranking points of each nation and the percentage of victories were used to estimate team performance. The study period was divided into sequences of 4 years corresponding to the period between two consecutive RWCs. For each sequence and nation, slopes and intercept of CSS trends were computed along with victory percentage and mean ranking points. Multiple linear regression analysis was used to establish the associations between team performance and experience.

Results: In regards to the CSS trends, both intra- and inter-nation variability appears to exist. Positive and negative slopes can be observed for the same team from one 4-year cycle to the next. Still, CSS slope is found to be significantly associated with both ranking points ( $p$ value $=0.042, R^{2}=0.13$ ) and victory percentage ( $p$ value $=0.001, R^{2}=0.42$ )

Conclusion: The evolution of the CSS that quantifies the collective experience of a team is linked to its performance. Such an indicator could be helpful in the decision-making process of national coaching staff.

Keywords: rugby, collective performance, collective efficacy, group dynamics, social networks

\section{INTRODUCTION}

Many studies have shown the importance of investigating cohesion and collective efficacy factors as key performance indicators in team sports (Heuzé et al., 2006; Bourbousson et al., 2010; Marcos et al., 2010; Leo et al., 2013; Fransen et al., 2015; Sedeaud et al., 2017). In rugby union, no significant effort can be performed without the help and cooperation of every player. In this context of cooperation, a team's performance can be described both by its productivity and by the sum of its players' abilities. Hence, the main goal of a coaching staff is to enable the team's performance to be greater than the simple sum of the parts. 
To do so, the coaching staff need to create cohesion and collective efficacy, the two have been proven determinants of performance. Qualitative aspects of collective experience on individual and collective performance have been explored. Heuzé et al. (2006) established that teammate's from more cohesive teams share stronger beliefs in their team. This enhances individuals' perception of collective efficacy thereby improving cohesion. In parallel, Marcos et al. (2010) added that improving team cohesion in basketball ameliorates a teammate's perception of efficacy. Moreover, Leo et al. (2013) stated that players with higher cohesion and collective efficacy profiles belonged to the best teams. Generating synergy through a coordinated effort which allows each member to maximize their strengths and minimize their weakness (Jain, 2009) is a long-term process (Sedeaud et al., 2017). It requires coordination based on shared knowledge and action (Eccles and Tenenbaum, 2004) that teammates can only achieve through time spent together and traditional methods of team-building (Shearer, 2015). Thus, impact of cohesion and collective efficacy gathered through collective experience has been widely qualitatively correlated with improvement of team performance.

Fewer studies have led to quantify player profiles and their potential relation on performance. Individual experience among the collective is a determinant for the team to perform. Previous studies have found that rugby union international players may be selected because of greater skill and experience (Walsh et al., 2007; Gabbett and Ryan, 2009; Hendricks and Lambert, 2014). Furthermore, the sum of individual experience among the squad has also been reviewed. Sedeaud et al. (2012) showed that teams which include forwards with previous World Cup experience perform better. Moreover, specific analysis on collective effectiveness of French national rugby team showed that more experienced forwards surround the best halfbacks, locks and centers (Sedeaud et al., 2017). Impact of player turnover rate on results has also been analyzed in rugby union and football. A high rate of turnover in the French national rugby team between two consecutive games is associated with the loss of the game (Sedeaud et al., 2017). Similar results have been found in football by Carling et al. (2015). The best results of one football team over 5 seasons were obtained when the fewest number of players were alternated over the season. This team won the national championship with the same 10 players involved in at least $75 \%$ of the total minutes played during the season. During the other seasons, $<6$ players had this characteristic of high playtime. This was potentially due to higher player availability and low injury incidence, which had a significant influence on team success (Hägglund et al., 2013). Those results imply that squad management strategies directly foster team performance.

The impact of team experience on team performance has been analyzed through qualitative estimators such as cohesion and collective efficacy, and then quantified by indicators summing individual experiences or assessing a global estimation of the group experience. The purpose of this study was to quantify team experience and its evolution through time, based on cumulative shared selections of players. Then, to assess its impact on team performance. As specified by Shearer (2015), the prestige and the 4 -year cycle between two Rugby World Cup events make this competition an ideal field of investigation. As shown in a recent paper from Mukherjee et al. (2019), we assume that a strong link exists between a team's history and its performance: that the greater the experience, the better the group's performance will be.

\section{METHODS}

\section{Data Collection}

After obtaining the approval of the National Institute of Sport, Expertise, and Performance ethics committee (Conseil Scientifique Medical et de Formation, CSMF), scoresheets of all games involving at least one of all 10 nations participating at the Rugby Championship (Argentina, Australia, New Zealand, and South Africa) and the Six Nations Championship (England, France, Ireland, Italy, Scotland, and Wales) were collected from the end of the 1999 Rugby World Cup (RWC) up to the 2015 RWC final. Date of the game, competition, points scored and conceded, names and positions (forwards and backs) of players involved as starters were recorded for each international game. Data were collected from an up-to-date rugby news and statistics website: www.statbunker.com. The site is updated after every game, and has a comprehensive list of statistics, covering all aspects of rugby union. Additional to the scoresheet data, World Rugby Ranking points of each nation were recorded weekly from its introduction at the beginning of 2004 up to the last game covered by our study period. Ranking points were collected on the World Rugby website: www.worldrugby.org.

\section{Team Experience Indicator: Cumulative Shared Selections}

First, we quantified team experience at a given game. A single indicator based on the set-lists of the starters selected by the head coach was produced. For each game, number of selections that each player has shared with the others during the previous games were computed and added up as follow.

Let's define for each national team a network with $n$ vertex corresponding to every players selected over $m$ matches through a certain period of time. Different vertex can or cannot be linked by edges $E$ depending on whether the players had played together at least once. Edges are weighted according to the number of match shared by the players: if two players $i$ and $j(i$ and $j \in\{1, \ldots, n\})$ play at a given match $k$, then $E_{i, j}^{k}=$ 1. If they don't or only one does play, then $E^{k}{ }_{i, j}=0$.

Hence, the number of CSS of a team at a given game $g(g \in\{1, \ldots, m\})$ is

$$
\operatorname{CSS}_{g}=\frac{1}{2} \times \sum_{k=1}^{g-1}\left(\sum_{i=1}^{n}\left(\sum_{j=1, j \neq i}^{n}\left(E_{i, j}^{k}\right)\right)\right) .
$$

This calculation quantifies the Cumulative Shared Selections (CSS) of a team at a given game. The number of CSS was computed over the entire period (1999-2015). Then we aimed to analyze the evolution of the team experience indicator through time. As implied by Shearer (2015), we chose to divide our study period into sequences of 4 years, each sequence corresponds to 


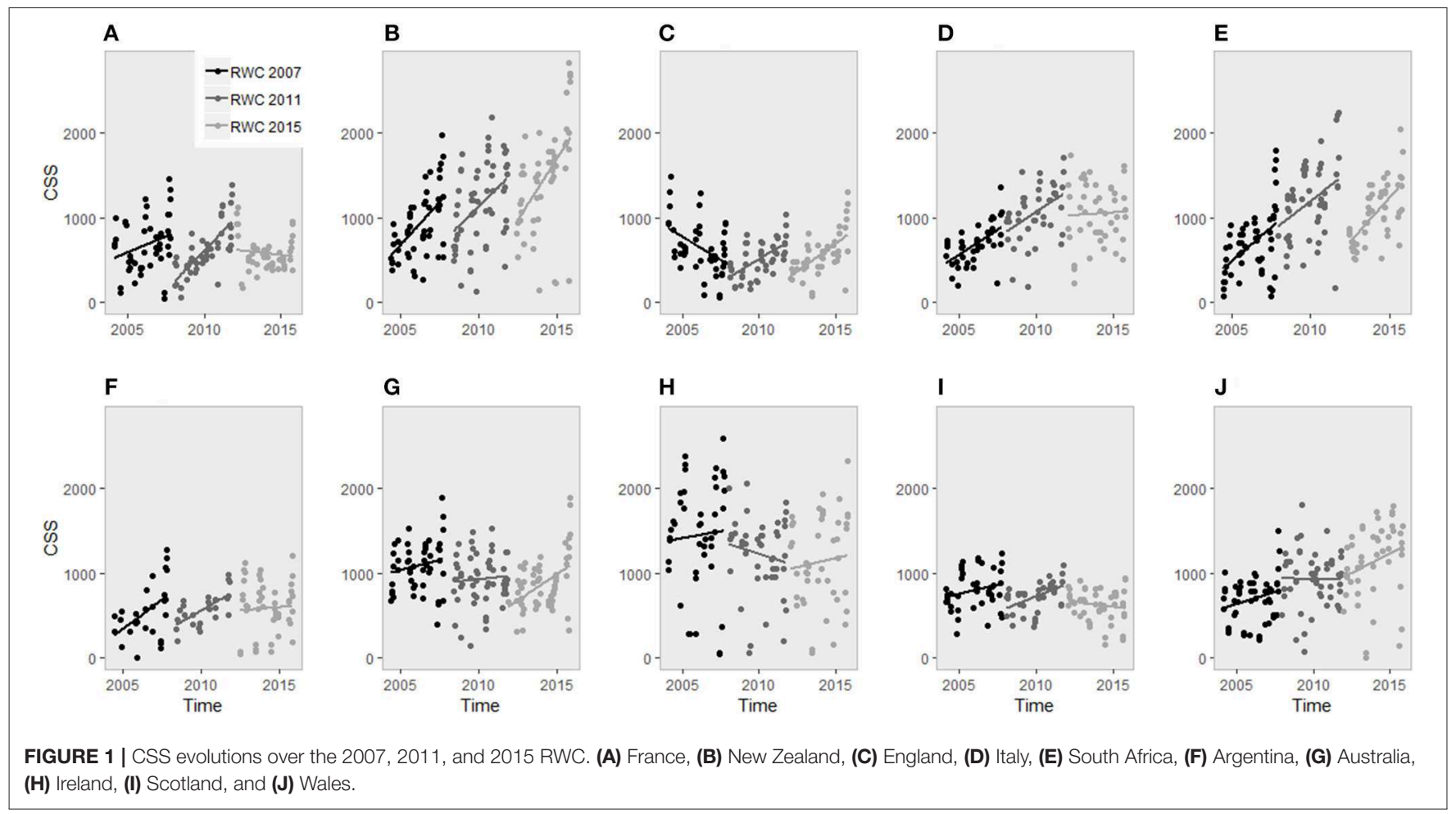

the period between two consecutive RWCs. Mean number of CSS was computed by nation for each sequence.

\section{Slope, Intercept, Ranking Points, and Victory}

The behavior of the team experience indicator through time was analyzed. Slopes and intercepts from linear regression between CSS's evolution and games played were computed and compared for each sequence and nation. The slope between two RWCs reflects how the CSS evolved in preparation for the following RWC. The intercept is used to for analysis adjustment over the basal team experience at the beginning of a RWC cycle.

Both World Rugby Ranking points and percentage of victories were used to estimate national team performance. From the same perspective of analyzing evolution of the team experience, mean ranking points and victory percentage were computed by nation for each 4 year time block.

\section{Statistical Analysis}

Data are reported as mean \pm standard deviation. Multiple linear regression analysis (see Table 1) is used for establishing the potential associations between team performance and team experience indicators which are entered as dependent variable. Please see below the multiple regression models:

$$
\begin{aligned}
\text { ranking points }= & \beta_{0}+\beta_{1} \times \text { CSS slope } \\
& +\beta_{2} \times \text { CSS intercept }+\varepsilon \\
\text { victory percentage }= & \beta_{0^{\prime}}+\beta_{3} \times \text { CSS slope } \\
& +\beta_{4} \times \text { CSS intercept }+\varepsilon^{\prime}
\end{aligned}
$$

Results are considered significant at $p<0.05$. All statistical analyses were performed using $\mathrm{R}$ (version 3.3.2; The $\mathrm{R}$ Foundation for Statistical Computing, Vienna, Austria).

Team experience estimated by the number of CSS computed in the first years of our study period is biased and does not take into account all previous games shared by the players. Therefore, specific analysis is conducted over three 4-year sequences between 2004 and 2015.

\section{RESULTS}

\section{Variable Descriptions}

The study period covered three sequences of 4 years between the $2003,2007,2011$, and 2015 RWCs. Mean number of games played by the 10 nations over a sequence is $45.9 \pm 6.9$, involving an average of $62.1 \pm 7.6$ different players. Regarding the experience indicators, mean number of CSS over a sequence is $860.4 \pm 284.9$ and it increases, on average, with a slope of $7.9 \pm 8.1$ and an intercept of $677.0 \pm 287.5$. Regarding performance indicators, the 10 nations completed the sequences with mean ranking points of $82.21 \pm 5.59$ and a mean victory percentage of $55.27 \pm 18.95$.

Figure 1 illustrates CSS evolutions over the 2007, 2011, and 2015 RWC for the 10 teams involved in four and six nations. Table 2 displays experience and performance indicators by nations for each sequence. Looking at England's trend of CSS, the slope leading to the first world cup is -9.03 resulting in a 2nd place ranking. Then, the slopes of the last two RWC period are 10.6 and 12.65 leading to a defeat in the quarter final and not progressing past the group phase, respectively. In the meantime, those slopes increase through the 3 periods and are 
TABLE 1 | Multiple linear regressions results

\begin{tabular}{lcccc}
\hline & Coefficient $(\boldsymbol{\beta})$ & SE & $\mathbf{9 5 \%} \mathbf{C l}$ & $\boldsymbol{p}$-value \\
\hline Ranking points & & & & \\
CSS slope & 0.36 & 0.16 & $0.074-0.643$ & 0.042 \\
CSS intercept & 0.005 & 0.005 & $-0.003-0.013$ & 0.330 \\
Victory percentage & & & & \\
CSS slope & 1.78 & 0.45 & $0.992-2.568$ & 0.001 \\
CSS intercept & 0.02 & 0.013 & $-0.005-0.04$ & 0.193 \\
\hline
\end{tabular}

Effects of team experience indicators over team performance.

associated with a $10 \%$ increase in victories per 4 -year sequence (from 44 to 64\%). In 2007, the RWC winner displayed a slope of 12.99 (Table 2). During the entire 12 years of follow-up, only Wales and New Zealand share a continuous progression of their CSS (Figure 1) and an uninterrupted improvement of victory percentage and ranking score (Table 2).

\section{Multiple Linear Regressions}

In multiple linear regressions, CSS slope is found to be significantly associated with both ranking points ( $p$ value $=0.042$, $\left.R^{2}=0.13\right)$ and victory percentage ( $p$ value $\left.=0.001, R^{2}=0.42\right)$, adjusted on the CSS intercept (Table $\mathbf{1}$ ).

\section{DISCUSSION}

This study is the first to create a collective performance indicator and reveal its association with ranking and victory.

We have investigated the 4-year cumulative shared selections evolution of the national rugby teams involved in four and six nations between consecutive RWCs from 2004 to 2015. A general indicator quantifying collective experience was computed. Its impact on rugby union game results was assessed. Previous work introduced the idea that shared selections are highly involved in rugby union teams' performance and that time is needed to let collective effectiveness emerge (Sedeaud et al., 2017). Sedeaud et al. concentrated their investigations on shared experiences between pairs of players (halfbacks, locks, or centers). Investigating shared experiences between all players reveals a new way to test these relationships.

Team cohesion and collective efficacy are widely used to qualify collective experience (Heuzé et al., 2006; Bourbousson et al., 2010). These qualities are acquired through the time players spent with each other (Shearer, 2015); CSS is proposed as a global statistical tool to quantify all the experiences shared between the players during successive games. By definition, this indicator changes after each game, making it a natural candidate to analyze team dynamic construction over a defined period. Slopes and intercepts of CSS evolution over 4-year cycles preceding RWCs put into numbers the strategies established by the coaching staff to create their teams.

\section{CSS Trends and Performance}

With regards to the CSS trends, its slopes and intercepts, both intra- and inter-nation variability appear to be wide. Positive and negative slopes can be observed for the same team from one 4year cycle to the next. For example, England presents opposite CSS evolutions leading up to the 2007, 2011, and 2015 RWCs with a negative slope followed by two positive ones. Those slopes are associated with a 10 percent increase in victories per 4-year sequence (from 44 to 64\%). On the other hand, South Africa seems to apply a different strategy for two successive RWCs, with two consecutive positive slopes (increasing team building), with the second one starting precisely where the first one ends, implying some continuity in the policy of player selection. Such a continuity observed for the 2011 RWC 4-year cycle could be explained by the confidence in the players after their success in the 2007 RWC. Nonetheless, a continuous improvement in CSS during a long period is paradoxical. It implies a general aging of the group which in reality is naturally topped out by the physical demands. Mean ages of RWC players of 25 years for backs and 27 for forwards (Sedeaud et al., 2012) reflect such a reality. The construction of a collective workforce that shares consequent playing time also faces the necessary youth physical prerequisites. Indeed, specific positions in rugby union demand repeated sprints, changes of direction, and capacities that generally decline with age (Berthelot et al., 2012; Marck et al., 2017).

Concerning multiple linear results exploring effects of experience indicators over performance ones, CSS slope is found to significantly impact both ranking points $\left(R^{2}=0.13\right)$ and victory percentage $\left(R^{2}=0.42\right)$ while CSS intercept does not. Despite the fact that a statistical relation between CSS slopes and team performance is highlighted, results must be interpreted cautiously. Only 13 and $42 \%$ of the observed variations can be explained by the model's inputs for ranking points and victory percentage, respectively. This remains an interesting result, which may imply that improving CSS (i.e., international games played together) over the entire 4 -year period prior to the RWC is more important that initial collective experience garnered in the past. In other words, progression is a key variable rather than the starting point. These results are consistent with previous conclusions showing that the same halfbacks, locks, or centers selected over time, obtained at the end of their common career, a winning percentage similar to the team's average (Sedeaud et al., 2017).

\section{CSS and Managing Strategies}

The rendez-vous of the RWC every 4 year allows to analyze how teams are built while preparing for such competitions (Shearer, 2015). For the best team, optimizing shared selections is a suitable strategy, but one that takes time and should be considered to have some limits. Furthermore, high intercepts are more often associated with negative or slight slopes: Australia, England in 2007, Ireland during the entire 12 years, and Italy in 2015. This reveals that the first year after an RCW may be crucial, that early choices of head coaches may be a primary determinant for the rest of the 4-year period (e.g., keeping attention to the incorporation process, mixing experienced players with young ones). New Zealand parallels these trends with a slight increase of intercepts, which results in a mix between players. This mix brings together experienced players, indispensable to the tactical group cohesion and optimal operation and 
TABLE 2 | Descriptions of the variables from the 10 nations over the 2007, 2011, and 2015 RWC.

\begin{tabular}{|c|c|c|c|c|c|c|c|}
\hline Nation & RWC & css & CSS slope & CSS intercept & Ranking points & Victory percentage & RWC result \\
\hline \multirow[t]{3}{*}{ New Zealand } & 2007 & $911.17 \pm 403.48$ & $16.59[9.61 ; 23.60]$ & 504.52 [307.71; 701.32] & $92.59 \pm 1.50$ & 87.5 & Quarter finalist \\
\hline & 2011 & $1,147.60 \pm 488.61$ & $14.39[6.98 ; 21.80]$ & 744.67 [506.22; 983.13] & $91.39 \pm 1.49$ & 83.64 & Winner \\
\hline & 2015 & $1451.26 \pm 632.65$ & $21.27[11.43 ; 31.11]$ & 876.95 [571.53; 1182.36] & $92.70 \pm 1.06$ & 94.12 & Winner \\
\hline \multirow[t]{3}{*}{ France } & 2007 & $675.49 \pm 324.91$ & $7.21[0.88 ; 13.54]$ & 495.33 [313.55; 677.11] & $85.18 \pm 1.26$ & 70.83 & Semi finalist \\
\hline & 2011 & $627.04 \pm 304.52$ & 18.20 [13.78; 22.62] & 208.42 [91.74; 325.10] & $82.02 \pm 1.91$ & 60,00 & Finalist \\
\hline & 2015 & $577.60 \pm 206.58$ & $-1.47[-6.29 ; 3.35]$ & 611.42 [484.21; 738.62] & $81.58 \pm 1.66$ & 46.51 & Quarter finalist \\
\hline \multirow[t]{3}{*}{ England } & 2007 & $645.06 \pm 309.81$ & $-9.03[-15.25 ;-2.81]$ & 861.73 [690.28; 1033.18] & $83.39 \pm 4.78$ & 44.68 & Finalist \\
\hline & 2011 & $515.53 \pm 212.82$ & $10.6[6.83 ; 14.37]$ & 271.83 [172.25; 371.42] & $82.33 \pm 1.5$ & 54.55 & Quarter finalist \\
\hline & 2015 & $561.18 \pm 259.9$ & $12.65[7.97 ; 17.33]$ & 270.23 [146.62; 393.84] & $84.24 \pm 1.57$ & 63.64 & Other \\
\hline \multirow[t]{3}{*}{ Italy } & 2007 & $679.85 \pm 238.3$ & $11.55[6.3 ; 16.79]$ & 437.35 [310.9; 563.81] & $72.81 \pm 1.44$ & 30,00 & Other \\
\hline & 2011 & $1,061.93 \pm 328.79$ & $11.72[4.01 ; 19.42]$ & 810.04 [619.93; 1000.16] & $73 \pm 1.08$ & 21.43 & Other \\
\hline & 2015 & $1,045.64 \pm 358.9$ & $1.07[-7.33 ; 9.47]$ & 1,021.04 [799.23; 1242.84] & $73.31 \pm 2.01$ & 24.44 & Other \\
\hline \multirow[t]{3}{*}{ South Africa } & 2007 & $684.96 \pm 386.84$ & $12.99[6.97 ; 19.01]$ & 334.24 [147.4; 521.08] & $85.74 \pm 1.97$ & 67.31 & Winner \\
\hline & 2011 & $1,194.94 \pm 483.66$ & $12.72[3.52 ; 21.92]$ & 876.93 [612.55; 1141.32] & $88.54 \pm 2.07$ & 63.27 & Quarter finalist \\
\hline & 2015 & $1,060.6 \pm 356.13$ & $16.97[11.34 ; 22.59]$ & 644.94 [486.61; 803.27] & $86.91 \pm 2.17$ & 69.57 & Semi finalist \\
\hline \multirow[t]{3}{*}{ Argentina } & 2007 & $543.71 \pm 331.87$ & $24.1[11.06 ; 37.14]$ & $194.28[-22.22 ; 410.78]$ & $79.29 \pm 3.53$ & 64.29 & Semi finalist \\
\hline & 2011 & $575.88 \pm 196.92$ & $18.34[9.07 ; 27.6]$ & 346.66 [214.24; 479.08] & $81.01 \pm 2.83$ & 37.5 & Quarter finalist \\
\hline & 2015 & $585 \pm 319.17$ & $2.44[-4.51 ; 9.39]$ & 526.34 [334.73; 717.95] & $77.72 \pm 2.58$ & 28.26 & Semi finalist \\
\hline \multirow[t]{3}{*}{ Australia } & 2007 & $1,085.35 \pm 293.76$ & $4.41[-1.48 ; 10.3]$ & 975.04 [805.79; 1,144.28] & $86.53 \pm 1.48$ & 60.42 & Quarter finalist \\
\hline & 2011 & $927.15 \pm 296.89$ & $2.22[-2.85 ; 7.29]$ & 864.91 [701.76; 1028.06] & $85.86 \pm 1.17$ & 59.26 & Semi finalist \\
\hline & 2015 & $874.39 \pm 320.51$ & $9.13[4.53 ; 13.73]$ & 609.64 [456.34; 762.95] & $86.28 \pm 1.81$ & 60,00 & Finalist \\
\hline \multirow[t]{3}{*}{ Ireland } & 2007 & $1,441.59 \pm 646.01$ & $4.49[-11.11 ; 20.09]$ & $1,340.61$ [937.62; 1743.61] & $82.27 \pm 1.56$ & 61.36 & Other \\
\hline & 2011 & $1,217.73 \pm 457.29$ & $-4.16[-14.79 ; 6.47]$ & $1,313.41[1032.61 ; 1594.22]$ & $81.01 \pm 2.3$ & 56.82 & Quarter finalist \\
\hline & 2015 & $1,133.61 \pm 543.61$ & $5.74[-7.31 ; 18.8]$ & $1,004.39$ [667.06; 1341.71] & $82.08 \pm 2.75$ & 61.9 & Quarter finalist \\
\hline \multirow[t]{3}{*}{ Scotland } & 2007 & $796.95 \pm 231.49$ & $5.43[-0.35 ; 11.2]$ & 680.22 [537.69; 822.76] & $75.34 \pm 1.31$ & 33.33 & Quarter finalist \\
\hline & 2011 & $724.92 \pm 171.38$ & $8.33[4.16 ; 12.5]$ & 558.28 [462.65; 653.92] & $76.98 \pm 1.87$ & 42.11 & Other \\
\hline & 2015 & $624.79 \pm 186.72$ & $-1.76[-5.68 ; 2.17]$ & $667.83[557.39 ; 778.26]$ & $76.49 \pm 1.22$ & 37.5 & Quarter finalist \\
\hline \multirow[t]{3}{*}{ Wales } & 2007 & $693.21 \pm 269.52$ & $5.35[-0.14 ; 10.84]$ & $562.16[407.68 ; 716.63]$ & $78.2 \pm 2.48$ & 45.65 & Other \\
\hline & 2011 & $931.72 \pm 332.22$ & $0.33[-6.29 ; 6.94]$ & 923.35 [729.58; 1117.12] & $79.43 \pm 1.89$ & 51.02 & Semi finalist \\
\hline & 2015 & $1,135.6 \pm 439.76$ & $8.76[-0.19 ; 17.71]$ & 921.03 [669.05; 1,173] & $81.65 \pm 2.17$ & 54.17 & Quarter finalist \\
\hline
\end{tabular}


progressively incorporates young players at the peak of their physical performance.

\section{Perspectives and Limitations}

The aim of this study was to quantify time spent together by the players and test whether a strong collective experience was correlated with better collective performance. Taking into account the fact that a squad can be composed of some players who play for the same club might be relevant. Groll and Abedieh (2013) showed in football that too many players coming from the same clubs negatively impacts the national selection's performance because of a lack of diversity (too many players scattered in too few clubs). In addition, it leads to a lack of knowledge of foreign game features. Future studies must investigate the impact of this factor in rugby union for comparison with results found by Groll in football. Comparisons between TOP 14 French players who come from many different clubs, with the New Zealand players involved in Super Rugby playing primarily for only 5 franchises could be relevant in order to identify different efficiency collective patterns.

By their construction and definition, team experience and performance indicators used in this study are macroscopic and unfortunately led to a loss of information (e.g., the victory percentage give no clue on the quality of games results). As a consequence of this limitation, some examples from our data do not necessarily support linear regression results. One can cite the negative CSS slope of England in 2007 leading them to an honorable 2nd place, or the strong slope (11.72) and intercept (810.04) of Italy associated to their lowest victory percentage (21.43). More accurate performance indicators must be taken in consideration in future analysis. Regarding team experience indicators, social network analysis (SNA) might help to provide deeper investigations. SNA techniques are increasingly applied to analyse behaviors within teams and inter-player interactions during games (Grund, 2012; Araújo and Davids, 2016; Cintia et al., 2016; Pina et al., 2017; Ribeiro et al., 2017). Bipartite structure of complex networks as described in Guillaume and Latapy (2004) might be an interesting tool to deeply scope the evolution of players' interactions through time and successive games, and might reveal individual or specific positions influencing the entire team evolution.

Due to a lack of information, we were not able to account for the impact of injuries on CSS evolution. The negative effect on team performance due to players unavailability caused by injuries has been shown in football (Parry and Drust, 2006; Hägglund et al., 2013; Carling et al., 2015; Windt et al., 2018). As

\section{REFERENCES}

Araújo, D., and Davids, K. (2016). Team synergies in sport: theory and measures. Front. Psychol. 7:1449. doi: 10.3389/fpsyg.2016.01449

Bengtsson, H., Ekstrand, J., and Hägglund, M. (2013). Muscle injury rates in professional football increase with fixture congestion: an 11-year follow-up of the UEFA Champions League injury study. Br. J. Sports Med. 47, 743-747. doi: 10.1136/bjsports-2013-092383 reported by Bengtsson et al. (2013), the influence of team rotation strategies on (a) team performance and (b) injury rates remains unclear. While our results shed light on the first relationship, further research must help to define the second one. Studying CSS impact on a team performance during a championship with large injury incidences (such as in rugby union) might help to better understand inherent relations between squad management, team performance and player injury.

\section{CONCLUSION}

For the first time, a single estimator (CSS) allows us to relate the evolution of a team's experience and its performance through time. This study reveals the potential of this indicator. It would be captivating to transpose this methodology to other team sports and other competition formats, such as a championship. Investigating more precisely the links between players through social network analyses would also make it possible to discretize relationships and detect key individuals or groups of individuals.

National coaching staff need to create the most competitive squad for each competition. They have to decide what to do with the time and few games to play that are given to them. The number of cumulative shared selections is a parameter that could help them in the decision-making process.

\section{DATA AVAILABILITY STATEMENT}

The datasets generated for this study are available on request to the corresponding author.

\section{AUTHOR CONTRIBUTIONS}

GS, JD, IM, JS, J-FT, and AS conceived, designed, performed, and analyzed the research. GS and AS wrote the manuscript. All authors read and approved the final manuscript.

\section{FUNDING}

This study was the result and part of a thesis (Saulière, 2018) funded by the National Institute of Sport, Performance and Expertise.

\section{ACKNOWLEDGMENTS}

We deeply thank Stacey Johnson and Stephanie Duncombe for reviewing the manuscript and providing language help. 
Carling, C., Le Gall, F., McCall, A., Nédélec, M., and Dupont, G. (2015). Squad management, injury and match performance in a professional soccer team over a championship-winning season. Eur. J. Sport Sci. 15, 573-582. doi: 10.1080/17461391.2014.955885

Cintia, P., Coscia, M., and Pappalardo, L. (2016). "The Haka network: evaluating rugby team performance with dynamic graph analysis," in 2016 IEEE/ACM International Conference on Advances in Social Networks Analysis and Mining (ASONAM) (San Francisco, CA: IEEE), 1095-1102.

Eccles, D. W., and Tenenbaum, G. (2004). Why an expert team is more than a team of experts: a social-cognitive conceptualization of team coordination and communication in sport. J. Sport Exerc. Psychol. 26, 542-560. doi: 10.1123 /jsep.26.4.542

Fransen, K., Decroos, S., Vanbeselaere, N., Vande Broek, G., De Cuyper, B., Vanroy, J., et al. (2015). Is team confidence the key to success? The reciprocal relation between collective efficacy, team outcome confidence, and perceptions of team performance during soccer games. J. Sports Sci. 33, 219-231. doi: 10.1080/02640414.2014.942689

Gabbett, T., and Ryan, P. (2009). Tackling technique, injury risk, and playing performance in high-performance collision sport athletes. Int. J. Sports Sci. Coaching 4, 521-533. doi: 10.1260/174795409790291402

Groll, A., and Abedieh, J. (2013). Spain retains its title and sets a new record - generalized linear mixed models on European football championships. J. Quant. Anal. Sports 9, 51-66. doi: 10.1515/jqas-2012-0046

Grund, T. U. (2012). Network structure and team performance: the case of English Premier League soccer teams. Soc. Networks 34, 682-690. doi: 10.1016/j.socnet.2012.08.004

Guillaume, J.-L., and Latapy, M. (2004). Bipartite structure of all complex networks. Inf. Process. Lett. 90, 215-221. doi: 10.1016/j.ipl.2004.03.007

Hägglund, M., Waldén, M., Magnusson, H., Kristenson, K., Bengtsson, H., and Ekstrand, J. (2013). Injuries affect team performance negatively in professional football: an 11-year follow-up of the UEFA Champions League injury study. $\mathrm{Br}$. J. Sports Med. 47:092215. doi: 10.1136/bjsports-2013-092215

Hendricks, S., and Lambert, M. I. (2014). Theoretical model describing the relationship between the number of tackles in which a player engages, tackle injury risk and tackle performance. J. Sports Sci. Med. 13, 715-717.

Heuzé, J.-P., Raimbault, N., and Fontayne, P. (2006). Relationships between cohesion, collective efficacy and performance in professional basketball teams: an examination of mediating effects. J. Sports Sci. 24, 59-68. doi: 10.1080/02640410500127736

Jain, N. (2009). "Build teams to run marathons, not sprints," in 97 Things Every Project Manager Should Know: Collective Wisdom From the Experts, ed B. Davis (Sebastopol, CA: O’Reilly Media, Inc.), 96.

Leo, F. M., Sánchez-Miguel, P. A., Sánchez-Oliva, D., Amado, D., and García-Calvo, T. (2013). Analysis of cohesion and collective efficacy profiles for the performance of soccer players. J. Hum. Kinet.39, 221-229. doi: 10.2478/hukin-2013-0085

Marck, A., Antero, J., Berthelot, G., Saulière, G., Jancovici, J.-M., MassonDelmotte, V., et al. (2017). Are we reaching the limits of homo sapiens? Front. Physiol. 8:812. doi: 10.3389/fphys.2017.00812
Marcos, F. M., Miguel, P. A., Oliva, D. S., and Calvo, T. G. (2010). Interactive effects of team cohesion on perceived efficacy in semi-professional sport. J. Sports Sci. Med. 9, 320-325.

Mukherjee, S., Huang, Y., Neidhardt, J., Uzzi, B., and Contractor, N. (2019). Prior shared success predicts victory in team competitions. Nat. Hum. Behav. 3, 74-81. doi: 10.1038/s41562-018-0460-y

Parry, L., and Drust, B. (2006). Is injury the major cause of elite soccer players being unavailable to train and play during the competitive season? Phys. Ther. Sport 7, 58-64. doi: 10.1016/j.ptsp.2006.03.003

Pina, T. J., Paulo, A., and Araújo, D. (2017). Network characteristics of successful performance in association football. A study on the UEFA champions league. Front. Psychol. 8:1173. doi: 10.3389/fpsyg.2017.01173

Ribeiro, J., Silva, P., Duarte, R., Davids, K., and Garganta, J. (2017). Team sports performance analysed through the lens of social network theory: implications for research and practice. Sports Med. 47, 1689-1696. doi: 10.1007/s40279-017-0695-1

Saulière, G. (2018). Analyse du Rapport Bénéfices/Risques et des Déterminants de la Performance dans les Sports Collectifs. Available online at: http://www.theses.fr/ 2018SACLS594 (accessed June 4, 2019).

Sedeaud, A., Marc, A., Schipman, J., Tafflet, M., Hager, J.-P., and Toussaint, J.-F. (2012). How they won Rugby World Cup through height, mass and collective experience. Br. J. Sports Med. 46, 580-584. doi: 10.1136/bjsports-2011090506

Sedeaud, A., Saulière, G., Marquet, L.-A., Del Vecchio, S., Bar-Hen, A., and Toussaint, J.-F. (2017). Collective effectiveness in the XV de France: selections and time matter. Eur. J. Sport Sci. 17, 656-664. doi: 10.1080/17461391.2017.1291742

Shearer, D. A. (2015). Collective efficacy at the Rugby World Cup 2015 - The role of imagery and observation. Eur. J. Sport Sci. 15, 530-535. doi: 10.1080/17461391.2015.1034787

Walsh, M., Young, B., Hill, B., Kittredge, K., and Horn, T. (2007). The effect of ball-carrying technique and experience on sprinting in rugby union. J. Sports Sci. 25, 185-192. doi: 10.1080/02640410600630571

Windt, J., Ekstrand, J., Khan, K. M., McCall, A., and Zumbo, B. D. (2018). Does player unavailability affect football teams' match physical outputs? A twoseason study of the UEFA champions league. J. Sci. Med. Sport. 21, 525-532. doi: $10.1016 /$ j.ptsp.2017.08.071

Conflict of Interest: The authors declare that the research was conducted in the absence of any commercial or financial relationships that could be construed as a potential conflict of interest.

Copyright (C) 2019 Saulière, Dedecker, Moussa, Schipman, Toussaint and Sedeaud. This is an open-access article distributed under the terms of the Creative Commons Attribution License (CC BY). The use, distribution or reproduction in other forums is permitted, provided the original author(s) and the copyright owner(s) are credited and that the original publication in this journal is cited, in accordance with accepted academic practice. No use, distribution or reproduction is permitted which does not comply with these terms. 